\title{
The Effect of Mouth Rinsing with Stabilized Chlorine Dioxide on Periodontitis
}

\author{
Shuichi Tsubura ${ }^{1,2, *}$, Kimio Uchiyama, ${ }^{2,3}$, Manabu Yamada ${ }^{2}$, Osamu Mimori², Shigeru Hiyama², Katsuma Katoh', \\ Masaki Shibata $^{2}$ \\ ${ }^{1}$ Department of Biochemistry, The Nippon Dental University School of Life Dentistry at Niigata, Hamaura-cho, Chuo-ku, Niigata, Japan \\ ${ }^{2}$ Department of Dentistry and Oral surgery, NHO Tochigi Medical Center, Nakatomatsuri, Utsunomiya, Tochigi, Japan \\ ${ }^{3}$ Clinical Research Department, NHO Tochigi Medical Center, Nakatomatsuri, Utsunomiya, Tochigi, Japan \\ *Corresponding author: tshu@ucatv.ne.jp
}

Received July 05, 2015; Revised July 26, 2015; Accepted August 02, 2015

\begin{abstract}
MATATACORO ${ }^{\mathrm{TM}}$ (containing $10 \mathrm{mg} / \mathrm{l}$ stabilized chlorine dioxide: MA-T, no alcohol, and is an odorless antibacterial agent) is useful for sterilization and disinfection. The aim of this study was to investigate whether MA-T is an appropriate mouth rinse for patients selected from whom undergoing initial periodontal treatment. The effect of rinsing with MA-T was compared with that with Concool $^{\mathbf{T M}}$ (containing $0.05 \%$ chlorhexidine gluconate: CX), Neosterine Green ${ }^{\mathrm{TM}}$ (containing $0.02 \%$ benzethonium chloride: NG), and purified water (PW). MA-T rinsing resulted in a marked change in the BANA-score and in the VAS scale. The mean BANA scores (score $\pm \mathrm{SD}$ ) on day 0 and day 30 were $1.55 \pm 0.50$ and $0.4 \pm 0.50(\mathrm{p}<0.001)$ for MA-T, $1.65 \pm 0.48$ and $0.75 \pm 0.44$ for CX, $1.43 \pm 0.50$ and $0.88 \pm 0.61$ for NG, and $1.83 \pm 0.38$ and $1.48 \pm 0.55$ for PW, respectively. The intraoral feeling caused by mouth rinsing was self-assessed using the VAS scores (mean \pm SD) on day 0 and day 30; they were $48.23 \pm 14.70$ and $74.43 \pm 7.33$ (26.2 $\mathrm{mm}$ increase) for MA-T, $50.45 \pm 11.94$ and $62.23 \pm 8.88$ (11.8 mm increase) for CX, 54.13 \pm 8.11 and $63.73 \pm 8.18$ (9.6 mm increase) for NG, and 54.70 \pm 6.06 and 59.18 \pm 6.11 (4.5 mm increase) for PW, respectively. These results suggest that MA-T induced marked reduction of periodontopathic oral bacteria and increased feeling of freshness after the treatment period. These results suggest that MA-T is an appropriate mouth rinse for patient with periodontitis.
\end{abstract}

Keywords: stabilized chlorine dioxide, mouth rinse, periodontitis, oral bacteria, freshness

Cite This Article: Shuichi Tsubura, Kimio Uchiyama, Manabu Yamada, Osamu Mimori, Shigeru Hiyama, Katsuma Katoh, and Masaki Shibata, "The Effect of Mouth Rinsing with Stabilized Chlorine Dioxide on Periodontitis.” Oral Surgery, Oral Medicine, Oral Radiology, vol. 3, no. 1 (2015): 20-24. doi: 10.12691/oral-3-1-5.

\section{Introduction}

Periodontitis, a chronic inflammatory disease of the tissues supporting the teeth, is caused by multiple factors, including oral bacteria, and is based on a person's genetic profile and environmental factors. [1] Therefore, there is no complete treatment technique for periodontitis. The first periodontal treatment is elimination of periodontopathic bacteria from the subgingival area. Oral hygiene guidance as part of general treatment is usually selected as the first-choice treatment. The general treatments for periodontitis consist of basically periodontal initial treatments, such as scaling, root planing, and periodontal surgery, or a combination of surgical and chemical methods including the use of antibacterial drugs, particularly amoxicillin, tetracycline, and azithromycin. $[2,3]$ However, the use of antibacterial drugs as the conventional treatment method is associated with a number of risks, including the production of drug-resistant bacteria and allergic reactions in elderly individuals [4].

On the other hand, it is well known that the use of mouth rinses has an effective and distinct role in the management of periodontitis both in the early stage and after general treatments. [5,6,7] The most popular products for mouth rinsing are those containing chlorhexidine gluconate or benzethonium chloride with alcohol. However, they have an unpleasant taste, their formulations have a harsh taste, they stain teeth, and they excessively stimulate the surface of the oral mucosa because of the high alcohol content. Therefore, alternative mouth rinses not containing alcohol are needed for the treatment of periodontitis.

The commercialized solution MATATACORO ${ }^{\mathrm{TM}}$ (MA$\mathrm{T}$ : ACENET Inc., Tokyo, Japan) containing $10 \mathrm{mg} / \mathrm{l}$ stabilized chlorine dioxide, which neither stains nor has a harsh taste, has antibacterial efficacy without alcohol. Stabilized chlorine dioxide is a compound with antimicrobial properties against not only oral bacteria but also a variety of bacteria. [8] Though oral rinsing with stabilized chlorine dioxide is expected to reduce the number of bacteria in the mouth, [9] there have been no reports on the clinical activity of this product.

This is the first clinical study to evaluate whether the oral administration of MA-T reduces the levels of periodontopathic bacteria. The effect of MA-T on periodontal inflammation was examined in chronic periodontitis patients. 


\section{Material and Methods}

\subsection{Patients and Study Design}

From January 2013 to November 2014, 160 subjects (80 males and 80 females; non-smokers; age, $46.4 \pm 15.0$ years (mean $\pm \mathrm{SD}$ ); age range $25-73$ years) with chronic periodontitis and no other severe general diseases were selected as participants. The inclusion criteria for applicants were: (1) having at least 20 natural teeth, and (2) at least eight teeth with probing periodontal pocket depth (PD) of $>4 \mathrm{~mm}$ on molar teeth. Teeth with excess mobility and/or abscess formation were excluded from the study.

All participants were selected from patients referred to the authors' clinics. The study protocol was approved by the Committee of Ethics Affairs of NHO Tochigi Medical Center and was conducted according to the principles outlined in the Declaration of Helsinki for experiments involving human subjects. The subjects provided their written, informed consent before participating in the 30day study and were confirmed to meet the following criteria: (1) received initial periodontal treatment, such as dental cleaning; (2) took no commercialized mouth-rinse within the previous month; and (3) took no antibiotic drugs within the previous month. Dental cleaning served as dental prophylaxis to remove all supra-gingival plaque, stain, and calculus before the study. After the start of this study, all participants did not received professional prophylaxis or additional brushing using ultrasonic toothbrushes or power toothbrushes.

A randomized, double-blind, placebo-controlled study design with four parallel groups was used. The subjects were separated randomly into four groups to receive MATATACORO $^{\mathrm{TM}}[\mathrm{MA}-\mathrm{T}](\mathrm{n}=40)$, Concool $^{\mathrm{TM}}$ (containing $0.05 \%$ chlorhexidine gluconate) [CX] $(n=40)$, Neosterine Green ${ }^{\text {TM }}$ (containing $0.02 \%$ benzethonium chloride) $[N G](n=40)$, and purified water [PW] group (n $=40$ ), respectively (Table 1 ).

Table 1. Distribution of study participants

\begin{tabular}{llll}
\hline Group & $\mathrm{N}$ & Sex & Age, years $($ mean $\pm \mathrm{SD})$ \\
\hline MA-T & 21 & Male & $46.4 \pm 15.0$ \\
& 19 & Female & $47.0 \pm 14.4$ \\
CX & 18 & Male & $48.3 \pm 13.2$ \\
& 22 & Female & $46.4 \pm 14.3$ \\
NG & 22 & Male & $45.4 \pm 15.2$ \\
& 18 & Female & $46.2 \pm 13.2$ \\
PW & 19 & Male & $44.9 \pm 15.5$ \\
& 21 & Female & $45.1 \pm 15.3$
\end{tabular}

After the initial periodontal treatment, mouth rinsing was done with MA-T, CX, NG, or PW (30 ml) for 30 seconds every morning and evening for 30 days. Tooth brushing was done three times per day without any toothpaste, and none of the teeth received any treatment during this study. All participants used standardized tooth brushes. During the study, participants kept their usual dietary habits for tooth brushing, but they were instructed to refrain from using any other commercial mouth rinses. Four anterior or premolar and molar teeth were selected for assessment of clinical parameters and subjected to microbial tests; these teeth had a pocket deeper than $4 \mathrm{~mm}$ and similar probing depths and gingival inflammation levels.

\subsection{VAS Scale}

The intra-oral feeling caused by mouth rinsing was selfassessed with the use of a Visual Analog Scale (VAS), in which the patients quantified their freshness response by making a mark on a 100-mm-long line that was anchored by word descriptors at each end: "unpleasant" at the left end and "very refreshed" at the right end. The patients marked the point on the line that described their perception of the feeling. Patients were asked: "How fresh do you feel today? Place a vertical mark on the line below to indicate how you feel today." The VAS scale was determined by measuring the distance in millimeters from the left-hand end of the line to the point that the patient marked.[10,11] Intra-oral feeling was measured for all of the mouth and was acquired by one clinician on day 0 , day 14 , and day 30. It was evaluated by the difference of VAS scores between day 0 and day 14 or day 30 .

\subsection{Clinical Parameters}

Probing Pocket Depth (PPD), Bleeding on Probing (BOP), and the Gingival Index (GI) were assessed for all participants on six sites per tooth at baseline (day 0), day 14, and day 30. PPD and BOP were assessed using a manual periodontal probe (CP10SE; HuFriedy, Chicago, IL, USA). For assessing PPD, a periodontal probe equipped with a 0.5 -mm-diameter tip was inserted into the gingival crevice and swept from the distal to the mesial aspect of the tooth at a depth of approximately $1 \mathrm{~mm}$ and an angle of approximately $60^{\circ}$ while maintaining contact with the sulcular epithelium. BOP was then recorded and scored as present/absent by running the probe $1-2 \mathrm{~mm}$ into the gingival crevice. Gingivitis of the buccal and lingual marginal gingiva and the interdental papillae of all scorable teeth was scored using the Loe-Silness GI on a 4point scale from 0 (absence of inflammation) to 3 (severe inflammation) [12].

\subsection{Bacterial Assay}

The BANA test (Knowell Therapeutic Technologies Inc., Toronto, Canada) is a chair-side diagnostic system that is highly sensitive and specific for determining the presence of "red-complex" periodontal pathogens (Porphyromonas gingivalis, Treponema denticola, and Tannerella forsythis). These bacteria have been implicated in periodontal disease and its progression.[13] The products of this reaction can be demonstrated by the color reaction on a reagent strip as a blue-black product. Assessments were made as the relative intensity of three categories of the blue-black color change (strong positive, positive, or negative). Plaque was collected for BANA analysis from the same tooth sites that were used for assessing the clinical parameters at day 0 , day 14 , and day 30. After incubation of plaques samples for $5 \mathrm{~min}$ at $35^{\circ} \mathrm{C}$ with Evan's black dye solution, naphthylamine, which is released as a result of the presence of any BANAhydrolyzing bacterial species, diffuses to form a permanent blue-black color. The relative intensity of the formed blue color (strong positive, positive, or negative) was assessed. 


\subsection{Statistical Analysis}

All data analysis was performed using the MannWhitney U-test with IBM SPSS statistics ver.19. Differences of $p<0.05$ were considered significant.

\section{Results}

A total of 160 participants with chronic periodontitis were selected according to the study design, and all participants completed the study. Between day 0 and day 30, mean difference of VAS scores were each 26.2mm(MA-T), 11.8mm(CX), 9.6mm(NG) and $4.5 \mathrm{~mm}(\mathrm{PW})$. MA-T was significantly more difference compared with PW. CX and NG were significantly more difference compared with PW. The improvement of VAS scores between day 0 and day 14 or day 30 are shown in Table 2. The mean PPD changes from day 0 to day 30 are shown in Table 3 . The BOP scores from day 0 to day 30 are shown in Table 4.The mean GI scores from day 0 to day 30 are shown in Table 5. PPD, BOP, and GI all decreased to day 30, but marked differences were not seen between MA-T and CX, NG, and PW, respectively. The mean BANA scores from day 0 to day 30 are shown in Table 6. MA-T was significantly more effective in lowering BANA scores at day $14(p<0.01)$ and day 30 $(p<0.001)$ than CX, at day $30(p<0.001)$ than NG, and at day $14(p<0.005)$ and day $30(p<0.001)$ than PW.

Table 2. Mean difference of VAS scale (scores \pm SD) with Matatacoro $^{\mathrm{TM}}, \mathrm{CX}, \mathrm{NG}$, and PW

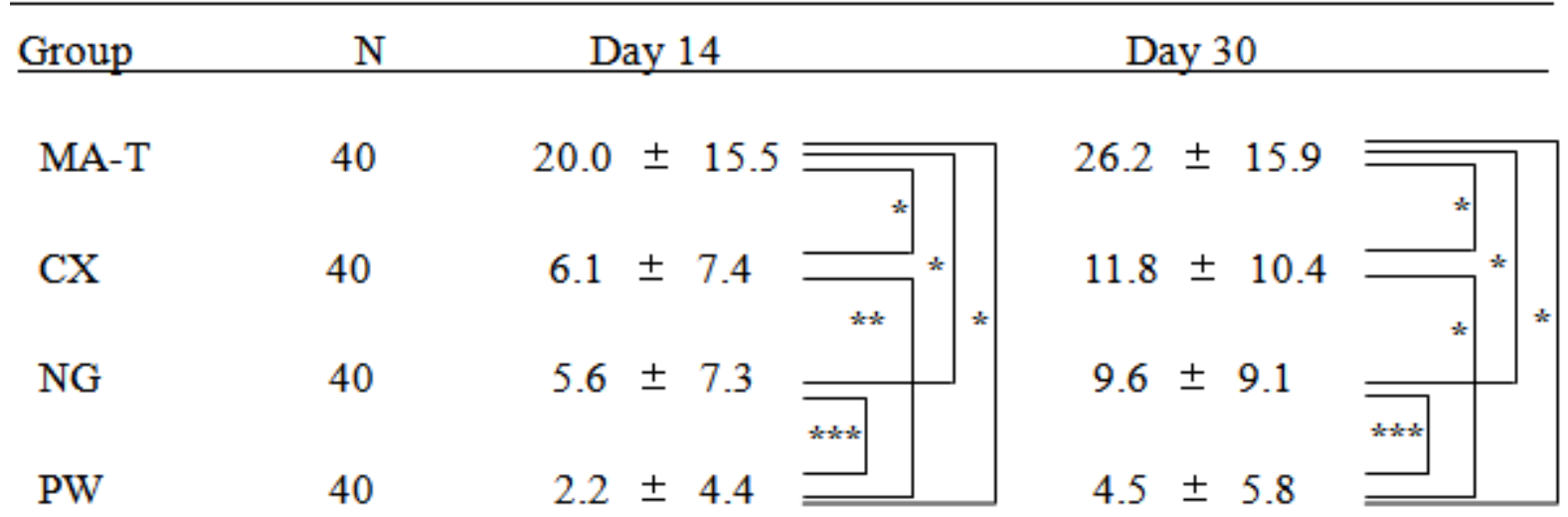

Given these results, MA-T appears to reduce periodontal oral bacteria after periodontal treatment and increases the feeling of freshness after the treatment period.

\section{Discussion}

In chronic periodontitis, it has been thought that maintaining adequate levels of oral hygiene using mechanical methods alone is impossible, and this has led to the adjunctive use of antimicrobial mouth rinses in oral hygiene regimens to help prevent and control chronic periodontitis [15]. Recently, 0.02\% chlorhexidine mouth rinse has become widely used in many countries. However, because of certain side effects associated with this agent, in particular allergic reactions, tooth staining, calculus formation, and taste aberrations, it was deemed desirable to use lower doses of this agent, if possible. Thus, there is a need for alternative mouth rinses for the treatment of periodontitis.

This was the first clinical study of the effects of stabilized chlorine dioxide (MA-T) on periodontitis in human subjects. It has neither a smell nor a harsh taste, and despite its mildness, which is similar to that of water, significant improvements in VAS scale scores were seen compared to other gargles. In this study, we estimated that MA-T has strongly improved on VAS score, and CX and NG also showed similar results. Each material has powers decreasing oral bacteria by long term use. However MA-T has neither smell nor harsh taste, and has especially the mildness those of similar to water, MA-T showed remarkable change on VAS score than the others. Thus, in totally, MA-T is more suitable mouth rinse than other materials. The mechanism, in which MA-T would cause a fresher taste or advantaged material, has not been unclear. It might to be that MA-T has neither smell nor harsh taste and are neutral $\mathrm{pH}$, and the change of BANA score indicated the target bacteria inside the periodontal pocket were decreased by the MA-T rinsing.

Robert et al. reported [16] that phosphate-buffered chlorine dioxide solution was effective in short-term trials for control of waterline contamination in ultrasonic dental scaling units. Furthermore, John et al. reported[17] that $0.04 \%$ stabilized chlorine dioxide, $3 \%$ sodium hypochlorite, and 2\% chlorhexidine were effective as endodontic irrigants in a polymicrobial biofilm. However, both studies used a bovine tooth model system, not human subjects in the clinical setting. From the beginning, stabilized chlorine dioxide was developed for dental units and ultrasonic scaler waterline contamination with potential pathogens such as alpha-hemolytic streptococci and some Gram-negative rods. [18,19,20,21] Stabilized chlorine dioxide solution is more favorable for some patients with periodontitis, better than other materials. MA-T has no objectionable pungent odor or bitter taste in its use as a lavage with ultrasonic scaler water line. Thus, it might be useful and effective for use as a mouth rinse. MA-T is expected to be more valuable for mouth rinsing in patients who have been treated for chronic periodontitis for long periods or who have xerostomia because it provides a weak stimulus and flavor.

The present results suggest that MA-T could be effective for improving conditions in the periodontal 
pocket and oral cavity of patients with chronic periodontitis. To minimize potential bias from this study, a double-blind, parallel, randomized, simple clinical study was performed, which included 160 subjects of both sexes ranging in age from 23 to 84 years. In order to confirm the results of the present study, further essential microbial experiments have to be performed on isolated periodontal pathogens; it is especially important to examine "redcomplex" pathogens in culture and monitor their growth in the presence of MA-T, CX, NG, and PW. Red-complex pathogens are among the most recognizable precursors initiating a cascade of inflammatory mediators, and an important change is the shift in the composition of microflora due to many factors, which results in microbial communities dominated by anaerobic bacteria.

Table 3. Mean Probing Pocket Depth $(\mathrm{mm} \pm \mathrm{SD})$ with Matatacoro ${ }^{\mathrm{TM}}$, CX, NG, and PW

\begin{tabular}{ccccc}
\hline Group & N & Day 0 & Day 14 & Day 30 \\
\hline MA-T & 40 & $5.00 \pm 0.75$ & $4.65 \pm 0.77$ & $4.48 \pm 0.75$ \\
CX & 40 & $4.90 \pm 0.71$ & $4.40 \pm 0.71$ & $4.03 \pm 0.70$ \\
NG & 40 & $4.53 \pm 0.68$ & $3.88 \pm 0.61$ & $3.50 \pm 0.51$ \\
PW & 40 & $4.90 \pm 0.59$ & $4.50 \pm 0.60$ & $4.38 \pm 0.59$ \\
\hline
\end{tabular}

Table 4. Mean Bleeding on Probing (BOP ${ }^{a}$ ) scores \pm SD with Matatacoro $^{\mathrm{TM}}, \mathrm{CX}, \mathrm{NG}$, and PW

\begin{tabular}{ccccc}
\hline Group & N & Day 0 & Day 14 & Day 30 \\
\hline MA-T & 40 & $1.63 \pm 0.49$ & $1.38 \pm 0.49$ & $1.13 \pm 0.52^{\mathrm{b}}$ \\
CX & 40 & $1.60 \pm 0.50$ & $1.15 \pm 0.53$ & $0.65 \pm 0.58^{\mathrm{b}}$ \\
NG & 40 & $1.45 \pm 0.50$ & $1.23 \pm 0.48$ & $0.88 \pm 0.56^{\mathrm{b}}$ \\
PW & 40 & $1.80 \pm 0.41$ & $1.50 \pm 0.55$ & $1.35 \pm 0.53$ \\
\hline
\end{tabular}

${ }^{\mathrm{a}}$ BOP scoring: 0 is non-bleeding to slight bleeding, tooth site of $0-30 \%$; 1 is moderate bleeding, tooth site of $31-60 \%$; 2 is severe bleeding, tooth site of more than $60 \%$.

b: Significant difference from day $0, \mathrm{p}<0.05$

The present results suggest that MA-T has the capacity to control chronic or acute periodontitis. Bacteriotherapy by mouth rinsing provides a more promising new research field of dental science than conventional oral health treatment. MA-T showed marked changes in the BANA test, but PPD, BOP, and GI did not show marked changes between MA-T and CX, NG, PW (Table 3-Table 5).

Table 5. Mean Gingival index $\left(\mathrm{GI}^{\mathrm{a}}\right)$ scores \pm SD with Matatacoro $^{\mathrm{TM}}$, CX, NG, and PW

\begin{tabular}{ccccc}
\hline Group & $\mathrm{N}$ & Day 0 & Day 14 & Day 30 \\
\hline MA-T & 40 & $1.65 \pm 0.74$ & $1.25 \pm 0.67$ & $1.05 \pm 0.68^{\mathrm{c}}$ \\
CX & 40 & $1.83 \pm 0.59$ & $1.28 \pm 0.60$ & $0.83 \pm 0.59^{\mathrm{c}}$ \\
NG & 40 & $1.65 \pm 0.74$ & $1.20 \pm 0.72$ & $0.88 \pm 0.61^{\mathrm{c}}$ \\
PW & 40 & $1.95 \pm 0.55$ & $1.55 \pm 0.55$ & $1.25 \pm 0.59^{\mathrm{c}}$ \\
\hline
\end{tabular}

${ }^{\mathrm{a}}$ GI scores: 0 is normal, 1 is slight gingival inflammation (no bleeding using instrument), 2 is moderate gingival inflammation (bleeding using instrument), 3 is gingival abscess, bleeding

c: Significant difference from day $0, \mathrm{p}<0.05$

In the present study, the effect of MA-T on periodontopathic oral bacteria was evaluated, and it was found that MA-T administration successfully decreased the numbers of periodontopathic bacteria in dental plaque after 30 days. MA-T is mildly effective for periodontal tissues, such as gingival epithelium and gingival capillaries. Thus, the mean PPD, BOP, and GI did not show marked changes. Otherwise, the mean BANA-score showed marked changes with MA-T, better than CX, NG, and PW. The target bacteria were significantly decreased after 30 days in the MT group (Table 6). It is likely that MA-T could play a greater role in situations requiring improvement of gingival crevicular fluid activity, although the mechanisms are still unclear . As the MA-T mouth rinse is a relatively simple treatment method, MA$\mathrm{T}$ is useful as a homecare material for preventing periodontal diseases. It is also well known that reduction of periodontal pathogens is important for clinical improvement and to decrease the risk of disease relapse. [14] Therefore, mouth rinsing with MA-T after initial treatment with periodontal mechanical debridement might represent an effective approach for the treatment of periodontitis.

Table 6. Mean BANA-score $^{\mathrm{a}} \pm$ SD with Matatacoro ${ }^{\mathrm{TM}}, \mathrm{CX}, \mathrm{NG}$ and PW

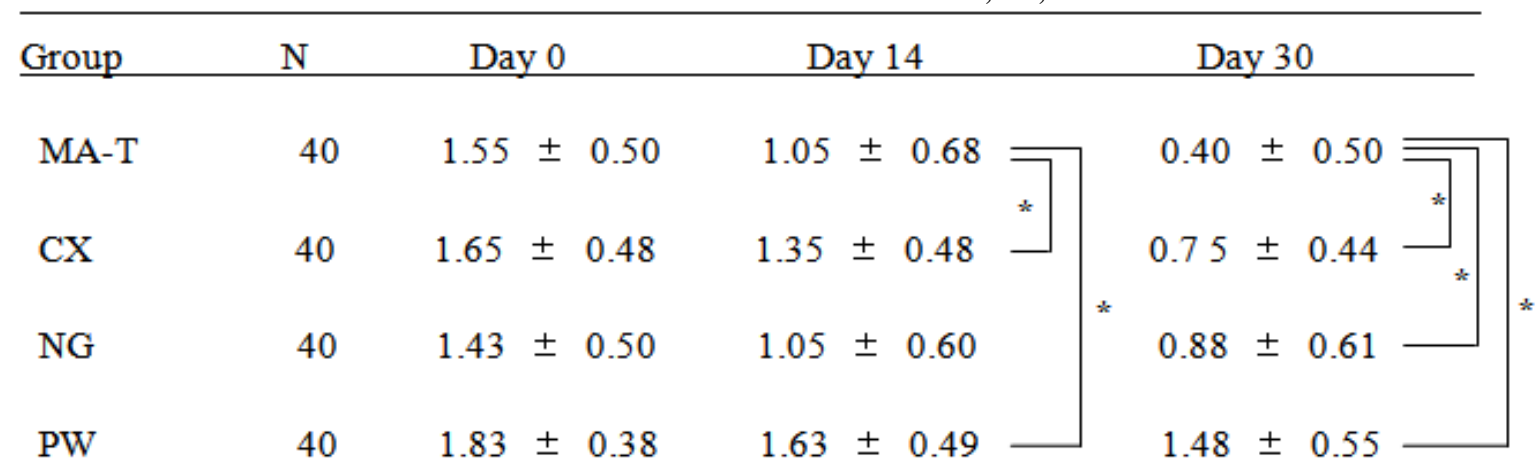

${ }^{a}$ BANA scores: 0 is negative, 1 is positive, 2 is strong positive

MA-T was significantly more effective with lowering BANA scores at day14 and day 30 compared with CX, at day 30 compared with NG, at Day14 and Day 30 compared with PW.

*: $p<0.05$

Therefore, more research is needed to identify appropriate effector strains for mouth rinsing specifically designed to prevent and treat periodontal disease. The present experimental protocol did not include any oral hygiene instruction before treatment or at baseline; however, subjects in all groups might have systematically altered their oral hygiene regimens due to the observation of routine dental work. Many factors, including attention bias, contribute to perceived placebo effects in clinical trials. However, this is an important first step, and one that provides valuable information as to relative levels of activity against oral bacteria in a very standardized laboratory setting. 


\section{Conclusions}

MA-T is an effective oral mouth rinse for patients with periodontitis. MA-T mouth rinsing could be useful for the treatment of inflammation and the clinical symptoms of periodontitis. The mechanism underlying the antibacterial effect of the MA-T mouth rinse is proposed to involve the inhibition of proteases that originate from oral bacteria.

Furthermore, MA-T mouth rinse may contribute to human health care with respect to not only oral diseases, but also the control of host immunological responses.

\section{Acknowledgments}

The authors would like to thank Dr Hiromi Shimomura for helpful and critical discussions.

\section{Statement of Competing Interests}

The authors have no competing interests.

\section{List of Abbreviations}

\author{
MA-T : MATATACORO ${ }^{\text {TM }}$ \\ $\mathrm{CX}:$ Concool $^{\mathrm{TM}}$ \\ NG : Neosterine Green ${ }^{\text {TM }}$ \\ PW : Purified water \\ VAS : Visual Analog Scale \\ PPD : Probing Pocket Depth \\ BOP : Bleeding on Probing \\ GI : Gingival Index
}

\section{References}

[1] Nishimura, F., Iwamoto, Y., Mineshiba, J. et al, "Periodontal disease and diabetes mellitus: the role of tumor necrosis factoralpha in a 2-way relationship," J Periodontol, 74(1). 97-102. Jan. 2003.

[2] Becker, W., Becker, B.E., Caffesse, et al, “A longitudinal study comparing scaling, osseous surgery, and modified Widman procedures: results after 5 years,” J Periodontol, 72(12). 1675-84. Dec. 2001.

[3] van Winkelhoff, A.J., Rams, T.E. and Slots, J., "Systemic antibiotic therapy in periodontitis," Periodontol 2000, 10. 45-78. Feb. 1996.

[4] Slots, J. and Rams, T.E., "Antibiotics in periodontal therapy: advantages and disadvantages," J Clin Periodontol, 17(7). 479-93. Aug. 1990.

[5] Ciancio, S.G., Lauciello, F., Shibly, O., et al, "The effect of an antiseptic mouthrinse on implant maintenance: Plaque and peri- implant gingival tissues,” J Periodontol, 66(11). 962-65. Nov. 1995.

[6] Christine, H., Charles, R.D.H., Naresh, C., et al, "Comparative efficacy of an antiseptic mouthrinse and an antiplaque/antigingivitis dentifrice," J Am Dent Assoc, 132(5). 670-75. May. 2001.

[7] Charles, C.H., Mostler, K.M., Bartels, L.L., et al, “Comparative antiplaque and antigingivitis effectiveness of a chlorhexidine and an essential oil mouthrinse: 6-month clinical trial,” J Clin Periodontol, 31(10). 878-84. Oct. 2004.

[8] Wirthlin, M.R., Ahn, B.J., Enriquez, B., et al, "Effects of stabilized chlorine dioxide and chlorhexidine mouthrinses in vitro on cells involved in periodontal healing," $J$ West Soc Periodontol Periodontal Abstr, 54(3). 67-71. 2006.

[9] Wirthlin, M.R., Choi, J.H. and Kye, S.B., "Use of chlorine dioxide mouthrinses as the ultrasonic scaling lavage reduces the viable bacteria in the general aerosols," $J$ West Soc Periodontol Periodontal Abstr, 54(2). 35-44. 2006.

[10] Holland, G.R., Narhi, M.N., Addy, M., et al, "Guidelines for the design and conduct of clinical trials on dentine hypersensitivity,” $J$ Clin Periodontol, 24(11). 808-13. Nov. 1997.

[11] Gould, D., Kelly, D., Goldstone, L., et al, "Examining the validity of pressure ulcer risk assessment scales: Developing and using illustrated patient stimulations to collect the data," J Clin Nursing, 10(5). 697-706. Sep. 2001.

[12] Loe, H. and Silness, J., "Periodontal disease in pregnancy. 1. Prevalence and severity,” Acta Odontol Scand, 21.533-51. Dec. 1963.

[13] Loesche, W.J., Bretz, W.A., Lopatin, D., et al, "Multi-center clinical evaluation of a chairside method for detecting certain periodontopathic bacteria in periodontal disease," $J$ Periodontol, 61(3).189-96. Mar. 1990.

[14] Haffajee, A.D., Cugini, M.A., Dibart, S., et al, "Clinical and microbiological features of subjects with adult periodontitis who responded poorly to scaling and root planning,” J Clin Periodontol, 24(10). 767-76. Oct. 1997.

[15] Anne, D.H, G. Torresyap, and Sigmund S.S, "Clinical changes following four different periodontal therapies for the treatment of chronic periodontitis: 1-year results," J.Clinic Periodontol, 34. 243-253. July. 2007.

[16] Robert, W., Grayson, W. and Marshall Jr., "Evaluation of ultrasonic scaling unit waterline contamination after use of chlorine dioxide mouthrinse lavage," J Periodontol. 72(3). 401-10. Mar. 2001.

[17] Lundstrom JR, Williamson AE, Vilhaver AL, et al. "Bactericidal activity of stabilized chlorine dioxide as an endodontic irrigant in a polymicrobial biofilm tooth model system,” J Endod, 36(11). 1874-78. Nov. 2010.

[18] Fitzgibbon, E.J., Bartzokas, C.A., Martin, M.V., et al, "The source, frequency and extent of bacterial contamination of dental unit water system,” Br Dent J. 157(3). 98-101. Aug. 1984.

[19] Karpay, R.I., Plamondon, T.J., Mills, S.E., et al, "Combining periodic and continuous sodium hypochlorite treatment to control biofilms in dental unit water systems," J Am Dent Assoc, 130(7). 957-65. Jul. 1999.

[20] Williams, H.N., Paszko-Kolva, C., Shahamat, M., et al, "Molecular techniques reveal high prevalence of Legionella in dental units,” J Am Dent Assoc, 127(8). 1188-93. Aug. 1996.

[21] Tippett, B.F., Edwards, J.L. and Jenkinson, H.F., "Bacterial contamination of dental unit water lines- a possible source of cross-infection,” NZ Dent J, 84(378). 112-13. Oct. 1988. 